\title{
Starch-based Carbon Nanotubes and Graphene: Preparation, Properties and Applications
}

\author{
Yun Chen, ${ }^{1,2}$ Zhanhu Guo, ${ }^{2, *}$ Rajib Das ${ }^{3}$ and Qinglong Jiang ${ }^{4, *}$
}

\begin{abstract}
Starch has received much attention due to the advantages, such as low cost, wide availability, and excellent compostability without toxic residues. However, starch-based materials are known with limitations related to weak mechanical properties and poor long-term stability, which could be overcome by incorporation with carbonaceous nanofillers. Thus, nanobiocomposites, namely dispersion of nano-sized fillers into a starch-biopolymer matrix, is one of the most promising technological advances. The starch-based carbonaceous nanofillers not only improve the poor mechanical properties of starch, but also change the performance of carbonaceous nanofillers solubility in water. This paper reviews the state-of-theart in the field of starch-based carbonaceous nanofillers. Two types of carbonaceous nanofillers including carbon nanotubes and graphene that have been used with starch are discussed. The main properties and applications of starch-based carbonaceous nanofillers are also discussed.
\end{abstract}

Keywords: Nanofiller, Starch, Composite.

Received:5 October 2020; Accepted: 2 November 2020.

Article type: Review article.

\section{Introduction}

Starch is the top two abundant polysaccharides, which have been used in food applications for years, such as sweetening, thickening, binding and emulsifying agents. ${ }^{[1]}$ As one of the main staples for a few countries, the abundant production of starch has a bright future in sustainable food supply. ${ }^{[2]}$ As the environment and the use of fossil resources are facing increasing challenges, therefore, starch used in non-food applications has experienced considerable development in the past decades. ${ }^{[3]}$ Like many other polymers, starch can be produced into different end-use forms such as extruded, molded, thermoformed, or blown articles. ${ }^{[4]}$ Starch-based materials still have limitations, such as poor processability and chemical modification. Starch-based biocomposites with

\footnotetext{
${ }^{1}$ Department of Food Science and Engineering, South China University of Technology, Guangzhou, 510006, China

${ }^{2}$ Department of Chemical \& Biomolecular Engineering, University of Tennessee Knoxville, Knoxville, TN 37996, USA

${ }^{3}$ Oxea Chemical company (OQ), Bay city, Texas 77414, USA

${ }^{4}$ Department of Chemistry and Physics, University of Arkansas, Pine Bluff, Arkansas 71601, USA

*Email: jiangq@uapb.edu (Q. Jiang), zguo10@utk.edu (Z. Guo)
}

improved performance have been developed in recent years..$^{[5-}$ ${ }^{7]}$ Nano-sized fillers which size range from $1 \mathrm{~nm}$ to $1000 \mathrm{~nm}$ are used to improve the performance and add new functionalities to starch-based materials.

As one of the carbonaceous nanofillers, carbon nanotubes (CNTs) display not only performance improvement but also new functionalities, especially electrical conductivity and electroactivity. ${ }^{[8,9]}$ However, the hydrophobicity of CNTs may limit their application. CNTs easily aggregated in the polymer matrix and resulted in poor reinforcing effects. Therefore, hydrophilic starch as a substrate can be combined with CNTs to improve the function of CNTs. Graphene, as another important carbonaceous nanofiller, has attracted strong scientific and technological attentions due to its great promise in many applications, such as electronics, energy storage/conversion, and bioscience/biotechnologies. ${ }^{[10-14]}$ Its excellent performance such as excellent thermal conductivity, electric conductivity and strong mechanical strength leads to its wide application. ${ }^{[15,16]}$ However, the inherent high specific surface area and wettability of graphene result in poor dispersibility and weak bonding with polymers which could limit the enhancement of both conductivity and mechanical properties. ${ }^{[17]}$ The efforts on the combination of graphene and natural resources as environmentally friendly alternatives to 
synthetic materials have attracted much attention. Starch with strong hydrophilicity can significantly improve the electrical and adsorption properties of graphene materials, which are hydrophobic and have low wettability.

Comparing with conventional synthetic polymers, starch has a unique chemical structure and processing behavior, and can be used as a composite biopolymer for many materials. Starch-based composite foams were prepared by compounding starch with PCL, chitosan, Latex, and PVOH. ${ }^{[18]}$ Nanocrystalline cellulose has been introduced as reinforcement for thermoplastic starch. ${ }^{[19]}$ According to Xie et al., various types of nanofillers that have been used with plasticized starch were discussed such as phyllosilicates and polysaccharide nanofillers. ${ }^{[3]}$ Thus, starch-based carbonaceous nanofillers can simultaneously improve the properties of starch and carbonaceous nanofillers, which can expand the application of starch and carbonaceous nanofillers. The incorporation of appropriately tuned carbonaceous nanofillers into starch as a biopolymer could provide complex structures and special properties. These nanocomposites with special properties could be designed for a wide range of conventional and emerging applications. This paper will provide a comprehensive review of starch-based carbonaceous nanofillers.

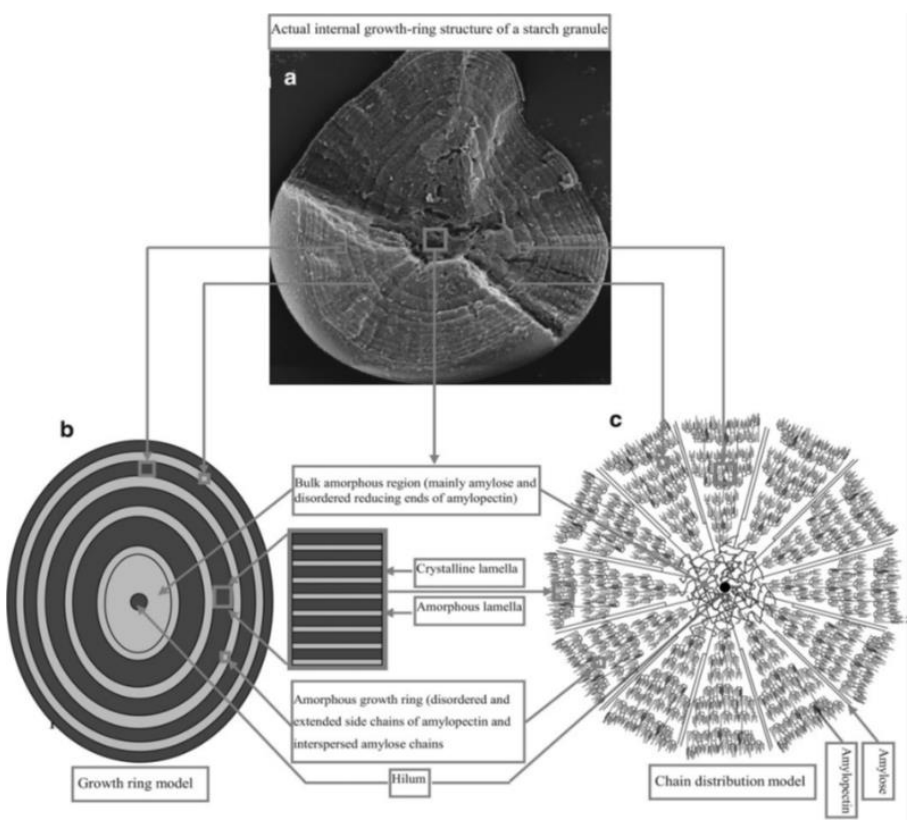

Fig. 1 Starch granule and its hierarchical structure (modified from a figure in Wang et al. ${ }^{[33]}$ )

\section{Granular and molecular structures of starch}

Starches which isolated from different botanical sources show characteristic granule morphology. ${ }^{[1]}$ The granule size, shape, crystallinity and internal molecular organization vary with the botanical source. Starch consists of two components: amylose and amylopectin. The two components together form alternating amorphous and semicrystalline shells (growth rings) (100-400 nm), crystalline and amorphous lamellae (periodicity) (9-10 $\mathrm{nm})$ and macromolecular chains $(\sim \mathrm{nm}){ }^{[1,20-22]}$ Normal native starches have $20-30 \%$ amylose and $70-80 \%$ amylopectin. Amylose is an essentially linear polymer which is formed by connecting $\alpha-1,4$ linked glucopyranosyl units. Amylopectin is a highly branched molecule that is formed by connecting $\alpha$-D-glucopyranosyl units in chains joined by a-1,6 linkages. Amylose has 5006,000 glucose units and molecular weight on the order of $10^{5}$ to $10^{6}$. Amylopectin, one of the largest molecules found in nature, has a molecular weight in the range of $10^{7}$ to $10^{9}$ and has 5000-50,000 D-glucose units which is much bigger than amylose. ${ }^{[23]}$ Within the starch granule, amylopectin molecules are oriented radially with the non-reducing ends of chains pointing toward the outer surface. Although the exact molecular architecture is still not clear, a cluster model for the structure of amylopectin was proposed by Robin et al. ${ }^{[24]}$ and it is widely used. This model is based mostly on indirect evidence from the analysis of amylopectin chain lengths, which does not indicate the distribution of the chains within the structure. In a more recent alternative model, the helical clusters are proposed to be arranged perpendicularly to a backbone formed from the long amylopectin chains. ${ }^{[1]}$

Starch granules are characterized by concentric growth rings originating from the hilum of the granule in the internal architecture. ${ }^{[25]}$ Each growth ring is composed of blocklets which consist of semi-crystalline lamellae. And the semicrystalline lamellae include crystalline lamellae which contain clusters of amylopectin chains in double helical configurations and amorphous lamellae which are considered mainly contain amylopectin branch points, amylopectin chains not organized into helical configurations, and long linear amylopectin chains that interconnect the clusters. ${ }^{[26]}$ The clusters of amylopectin helices have A, B and C type crystalline structures. The A-type crystal structures, which are more densely packed occur mainly in cereal starches and B-type structures, are predominant in tuber, root and high-amylose starches. $\mathrm{C}$ type is the mixture of A- and B-type crystals, which occurs in native starch from legumes, some roots and fruits. ${ }^{[27-29]}$ In most studies, B-type crystalline polymorphs are proposed to be in the center of the granules surrounded by peripheral A-type polymorphs, whereas the opposite occurs in high-amylose mutant rice starch. The cluster structure of amylopectin has a significant effect on the thermal properties and digestibility of starch. ${ }^{[30,31]}$

The amorphous regions and the location of amylose in the granules are not clear. There are various hypotheses: some studies showed that amylose chains were involved in interactions with amylopectin and more concentrated at the periphery than at the core of the granules; ${ }^{[32]}$ some hypotheses indicated that amylose molecules formed the bulk of the amorphous core of granules and a small number of amylose chains interspersed among the amylopectin clusters oriented towards the outer surface of the granules act as reinforcing rods. ${ }^{[33]}$

\section{Starch-based carbon nanotubes}




\subsection{Carbon nanotubes}

CNTs are unique tubular structures that have nanometer diameter and large length/diameter ratio. CNTs consist of one to hundreds of concentric shells of carbons with adjacent shells separation of $0.34 \mathrm{~nm}$. The carbon network of the shells is closely related to the honeycomb arrangement of the carbon atoms in the graphite sheets. The amazing mechanical and electronic properties of the nanotubes stem in their quasi-onedimensional (1D) structure and the graphite-like arrangement of the carbon atoms in the shells. Thus, the nanotubes have high Young's modulus and tensile strength, which makes them preferable for composite materials with improved mechanical properties. CNTs include single-wall carbon nanotubes(SWCNTs) and multi-wall carbon nanotubes (MWCNTs). Despite the wide use of CNTs in other polymer nanocomposite systems, ${ }^{[34]}$ the use of CNTs as the nanofiller for starch-based materials has just been initiated and MWCNTs were more widely used than SWCNTs. This may be due to the lower price and more abundance of MWCNTs than SWCNTs. Besides, MWCNTs exhibit a high aspect ratio $(\sim 1000)$, excellent mechanical, thermal and electrical properties.

\subsection{Preparation starch-based carbon nanotubes}

There are two main forms of compounding starch and CNTs, which include convenient solution process assisted mixing and casting method, the other way is CNTs functionalized by starch. In the first method, much care should be taken to choose the sequence of addition of ingredients which might affect the nanofiller dispersion, the gelatinisation/plasticisation, and thus the final structure and properties of the nano-biocomposite. This method for preparing starch-CNTs composites is simple physical mixing, and therefore strong chemical bonds such as H-bonds are not formed between starch and CNTs. The starch functionalized CNTs, or CNTs modified starch generally require the pretreatment of starch and CNTs to functionalize the surface. After providing more functional groups to the surface of starch, a tighter(robust) nanocomposite is formed between starch and CNTs.

\subsection{Properties and applications of starch-based carbon nanotubes}

After compounding starch with CNTs, some excellent properties and wide applications will appear. According to Cheng et al. starch-based carbon nanotubes films based on plasticized starch and modified-carbon nanotubes were prepared by a simple casting method. ${ }^{[35]}$ CNTs were oxidized to prepare CNT oxide (OCNT) by Hummer's method, and OCNTs were reduced by glucose to obtain reduced CNT (RCNT). The thermogravimetric results revealed that OCNTs and RCNTs contained about 15 and $8 \mathrm{wt} \%$ oxygen containing groups respectively, which made them disperse well in water ${ }^{[35]}$ Therefore, the plasticized starch-CNT, plasticized starch-OCNT, and plasticized starch-RCNT composites could be used to fabricate composites by casting process easily. The tensile strength, moisture resistance and electrical conductivities of the composites were tested in this study. The TEM images of the CNTs, OCNTs and RCNTs are shown in Fig. 2. They found that the reinforcing effect of RCNTs was more obvious than CNTs. The moisture resistance of CNTs was better than that of OCNTs and RCNTs. The electrical conductivities of plasticized starch-CNT, plasticized starchOCNT, and plasticized starch-RCNT composites were no obvious difference.
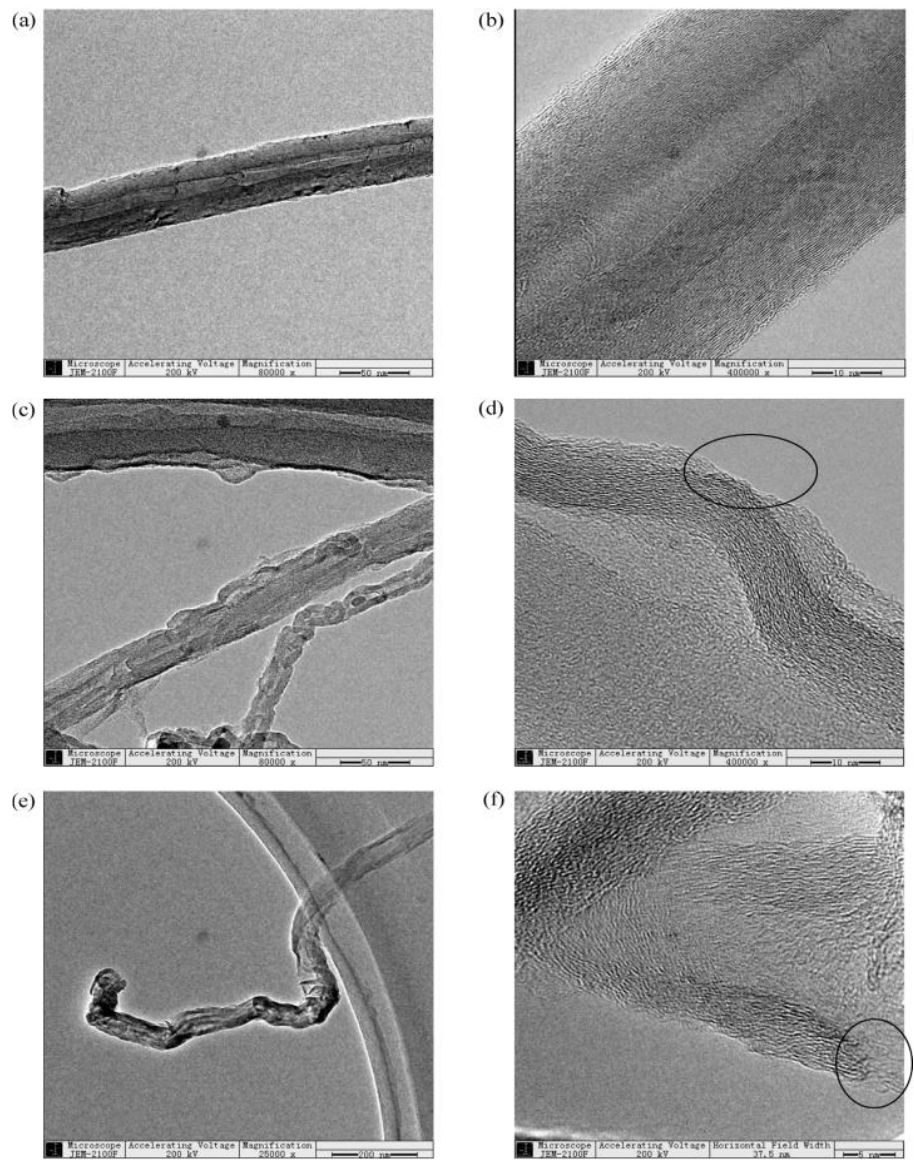

Fig. 2 TEM of CNT ( $a$ and b), OCNT (c and d), and RCNT (e and f). Reprinted from. ${ }^{[35]}$

Nanocomposite films were prepared from plasticized starch/functionalized multiwalled carbon nanotubes by a simple solution casting method to make as a gas barrier ${ }^{[36]}$ The multiwalled carbon nanotubes were functionalized by the hydrogen peroxide and $\mathrm{H}_{2} \mathrm{SO}_{4}$ and then mixed with plasticized starch by casting method. The thermal conductivity was enhanced after starch incorporation of a small amount of functionalized multiwalled carbon nanotubes. The electrical conductivity of starch/ functionalized multiwall carbon nanotubes was also increased which may be due to the good connectivity of carbon nanotubes. Cao et al. also prepared nanocomposites from plasticized starch and multiwall carbon nanotubes by a simple method of solution casting and evaporation. ${ }^{[37]}$ The morphology, thermal behavior, and mechanical properties of the films were investigated in their 
study. After MWCNTs introduced into the plasticized starch, the tensile strength and Young's modulus of the nanocomposites were enhanced significantly from 2.85 to 4.73 Mpa and from 20.74 to $39.18 \mathrm{MPa}$, respectively with an increase in MWCNTs content from 0 to $3.0 \mathrm{wt} \%$, respectively. The value of elongation at break of the nanocomposites was higher than that of plasticized starch and reached a maximum value as the MWCNTs content was at $1.0 \mathrm{wt} \%$. Besides the improvement of mechanical properties, the incorporation of MWCNTs into the plasticized starch matrix also led to a decrease in the water sensitivity of the plasticized starch-based materials. Famá et al. reported increments up to almost $70 \%$ in stiffness and $35 \%$ in ultimate tensile strength, and keeping deformations higher than $80 \%$ without break after starch films bound with very small quantities of multi-walled carbon nanotubes. ${ }^{[38]}$ Therefore, tensile toughness also increased by up to $\sim 50 \%$. Enhancements up to $\sim 100 \%$ in biaxial impact parameters were also observed. In addition to assisted mixing and casting, starch can be grafting onto the surfaces of functionalized multiwalled carbon nanotubes. ${ }^{[39]}$ FTIR revealed that the covalent bonds between $-\mathrm{OH}$ groups of soluble starch and carboxylated MWCNTs. TEM and TG showed that carboxylated MWCNTs were covered with the grafted starch. The grafted starch facilitated the dispersion of carboxylated MWCNTs-starch in water and chitosan films because of the hydrophilic of starch. As for electrochemical properties, compared to carboxylated MWCNTs, carboxylated MWCNTs-starch exhibited a couple of redox peaks in cyclic voltammograms testing.

In the process of compounding starch with CNTs, other nanomaterials can be added to improve the performance of the composite material. A novel nanocomposite material with electrochemical sensing was prepared from polyaniline/multiwall carbon nanotubes/starch as a good electrode material. ${ }^{[40]}$ The developed ternary composite system has manifold interactions and synergistic improved propertieshigh surface area, good electro-activity, stable dispersion, biocompatible, hydrophilic, multifunctional, and nanoturmeric shape morphology. Moreover, the polyaniline/multiwall carbon nanotubes/starch composites were fabricated for hydrogen peroxide biosensor with hemoglobin. The molecular structures of MWCNTs, starch, polyaniline, and hemoglobin were shown in Fig. 3. The developed biosensor showed a linear range result, the limit of detection, sensitivity, and long-term storage and stability. This material could be used as a platform to develop some other sensors by using other redox enzymes.

Functionalized multiwalled carbon nanotubes were used as reinforcing agents for poly(vinyl alcohol)/starch nanocomposites. ${ }^{[4]}$ MWCNTs were carboxylated by a conventional acid oxidation process and then poly(ethylene glycol) monomethylether was covalently attached to the carboxylated MWCNTs via esterification. The chemical structures of all samples as representatives were characterized by Fourier transform infrared spectroscopy and the morphology of the nanocomposites was probed by scanning electron microscopy. Moreover, the mechanical properties of the nanocomposites were improved due to the presence of functionalized MWCNTs. In conclusion, these materials show great potential in further applications in the coming decades and become an alternative engineering thermoplastic due to the processability, biodegradability, and acceptable cost. Polymer nanocomposites based on poly(vinyl alcohol)/starch blend and carbon nanotubes (CNT) were also prepared according to Jose et al. ${ }^{[42]}$

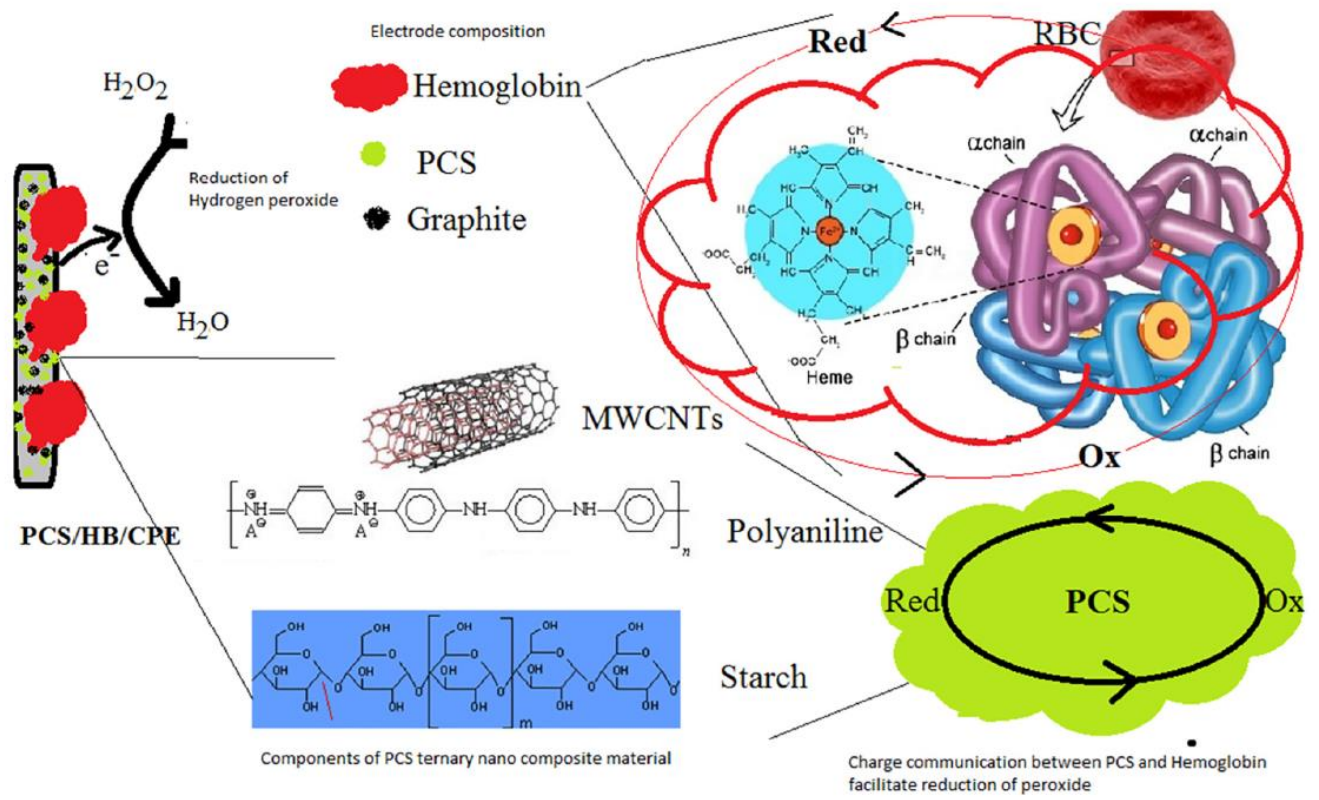

Fig. 3 Schematic diagram of functioning of peroxide biosensor, and molecular structures of MWCNTs, starch, polyaniline, and hemoglobin. Reprinted from. ${ }^{[40]}$ 


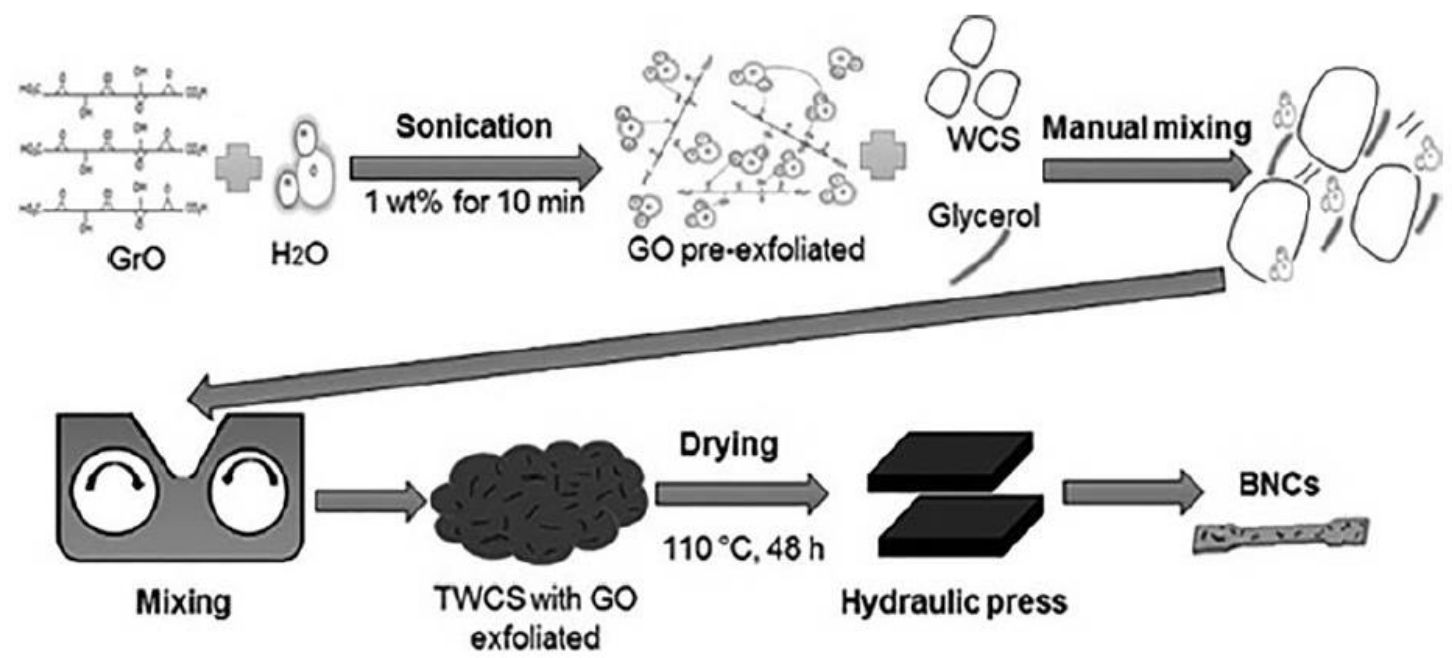

Fig. 4 Preparation scheme of the thermoplastic starch and graphene oxide bionanocomposites. Reprinted from. ${ }^{[51]}$

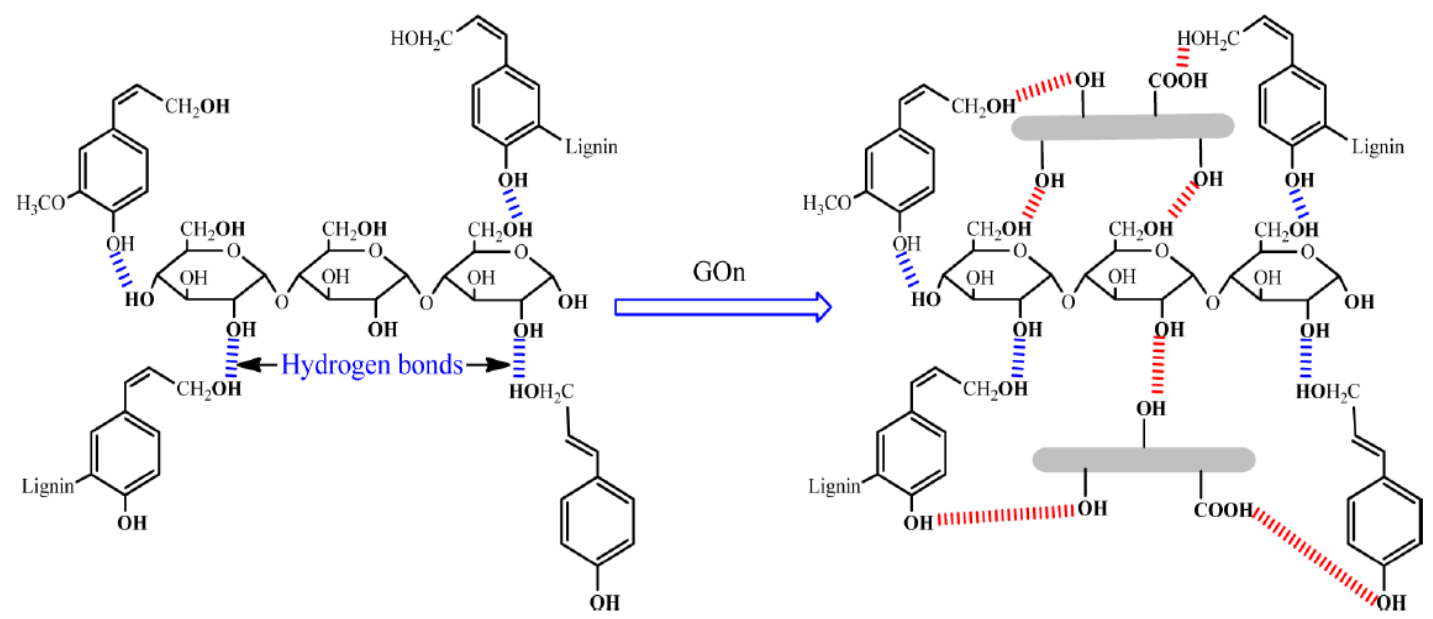

Fig. 5 Schematic representation for the structure of the starch/lignin-GO bionanocomposite film and the possible interactions between starch, lignin, and GO nanosheets. Reprinted from. ${ }^{[54]}$

CNTs were added to the polycaprolactone and as a template for the growth of iron oxide nanoparticles which thermoplastic starch to prepared nanocomposites. A were uniformly dispersed on the surface of the MWCNTpolycaprolactone/CNT masterbatch was first prepared and starch. MWCNT-starch-iron oxide exhibited then blended with $20 \mathrm{wt} \%$ thermoplastic starch. The results of superparamagnetic properties with a saturation magnetization the transmission and SEM images reveal a CNTs localization principally in the thermoplastic starch phase and partly at the polycaprolactone/thermoplastic starch interface, which indicates a strong driving force for the CNTs toward thermoplastic starch. The addition of CNTs to polycaprolactone resulted in an increase in the crystallization temperature and a decrease in the percent crystallinity, confirming the heterogeneous nucleating effect of the nanotubes. Finally, DMA analysis revealed a dramatic decrease in the starch-rich phase transition temperature $\left(\sim 26{ }^{\circ} \mathrm{C}\right)$, for the system with nanotubes located in the thermoplastic starch phase. Multiwall carbon nanotube composites functionalized by soluble starch were used to adsorb dyes in the study by Chang et al. ${ }^{[43]}$ The results of the study showed that the starch component (about $14.3 \mathrm{wt} \%$ ) was covalently grafted onto the surface of MWCNT. Starch acted (23.15 emu/g) and better adsorption for anionic methyl orange and cationic methylene blue dyes than MWCNT-iron oxide.

\section{Starch-based carbon nanotubes graphene 4.1 Graphene}

Graphene, a single-layer carbon sheet with a hexagonal packed lattice structure, has attracted tremendous research interest in recent years. It has exceptional properties, such as the quantum hall effect, high carrier mobility at room temperature, large theoretical specific surface area, good optical transparency, high Young's modulus and excellent thermal conductivity. ${ }^{[4-46]}$ The scaled-up and reliable production of graphene derivatives, such as graphene oxide (GO) and reduced graphene oxide (rGO), offers a wide range of possibilities to synthesize graphene-based functional materials for various applications. To date, graphene-based 
composites have been successfully made with inorganic nanostructures, polymers, and so on. ${ }^{[47,48]}$ While graphenebased composites cannot be dispersed in water or organic solvent, which limited the use of graphene, graphite oxide (GO) is hydrophilic and can form strong physical interactions with a polymer like starch due to its various oxygen functional groups including hydroxyls, epoxides, carbonyls, and carboxyls.

\subsection{Preparation starch-based graphene}

There are two main forms of compounding starch and graphene: convenient solution process assisted mixing and casting method; and graphene oxide reduced by soluble starch. The soluble starch is the starch powder from food products. This kind of starch has similar structure as glucose, thus, it has nearly the same reductive ability as glucose for graphene oxide reduction. ${ }^{[49]}$ After a reaction between starch with more functional groups and graphene oxide, a tighter nanocomposite is formed in the process of compounding starch with graphene, other nanomaterials can be added to improve the performance of the composite material such as poly (vinyl alcohol) and lignin. In addition to prepare a film, the composite of starch and graphene can also form hydrogels, which provide cross-linked network.

\subsection{Properties and applications of starch-based graphene}

The casting process was used to prepared RGO/plasticizedstarch. ${ }^{[50]}$ The abundant residual oxygen-containing groups of RGO could form hydrogen bond interactions with starch. These interactions and the unidirectional/uniform dispersion of RGO sheets in the plasticized-starch matrix played important roles in improvements to mechanical and moisture barrier properties. The RGO/PS (polystyrene) composites with desirable mechanical properties are some of the most promising candidates for advanced UV shielding, biochemical, or electrochemical materials. Waxy corn starch, glycerol, and graphene oxide were used to prepare nanocomposites. ${ }^{[51]}$ The preparation scheme is showed in Fig. 4. The results showed that this bionanocomposite had an irregular texture, a good dispersion of GO, and higher thermal stability than thermoplastic starch. The tensile strength and Young's modulus increased by $140 \%$ and $230 \%$ at a GO loading level of $0.5 \%$ due to good interfacial interactions of $\mathrm{GO}$ and the waxy corn starch matrix.
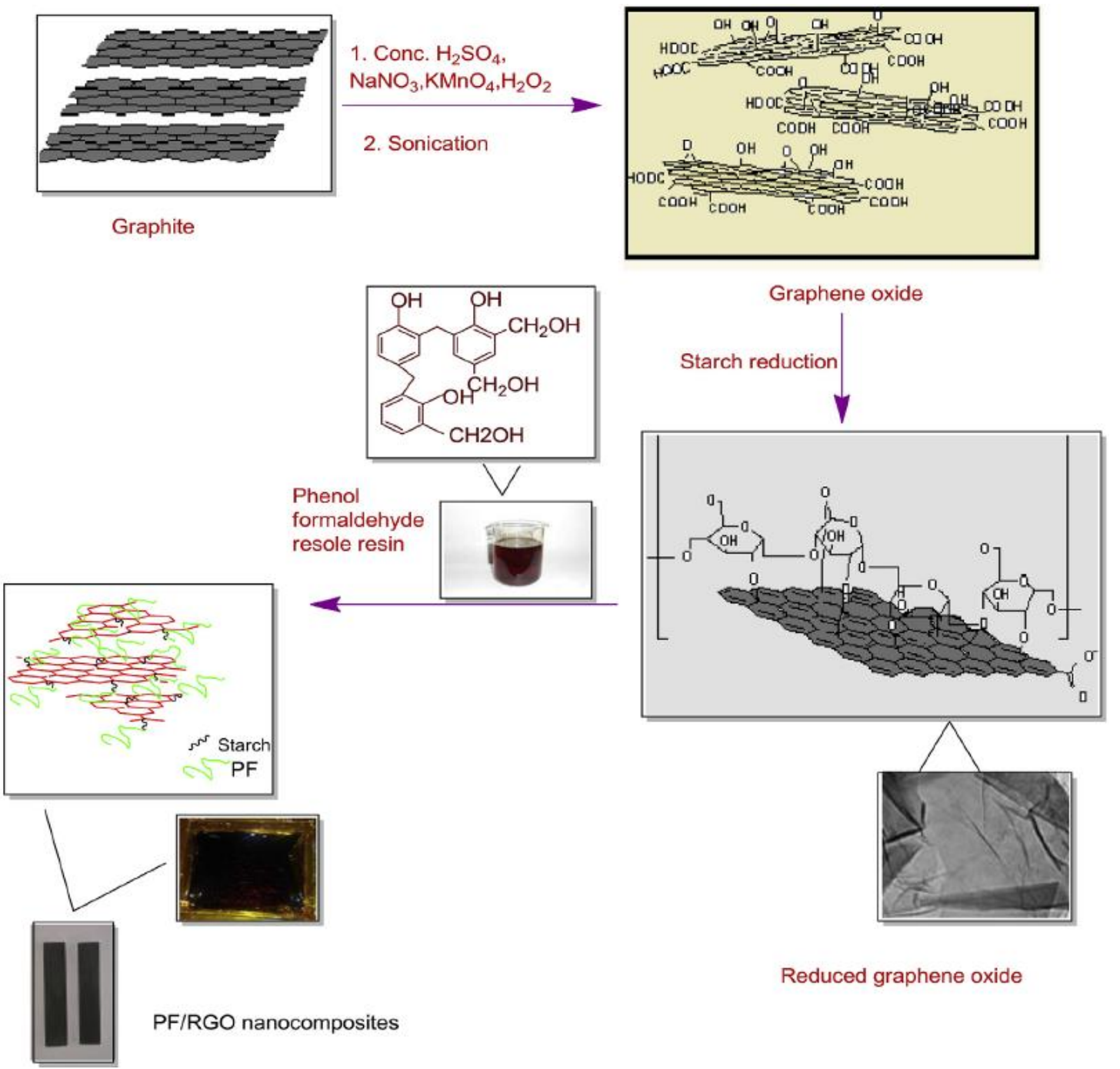

Reduced graphene oxide

Fig. 6 Schematic representation of the preparation of PF/RGO polymer nanocomposite. Reprinted from. $^{[56]}$ 
In the process of compounding starch with graphene, other nanomaterials can be added to improve the performance of the composite materials. Acetylated starch and poly (vinyl alcohol) loaded with graphene oxide were used to prepared bionanocomposite films. ${ }^{[52]}$ The results of the analysis showed that molecular-level interactions between components are mainly hydrogen-bonding types. After the photochemical treatment, the thermal diffusivity and electrical resistivity of starch-GO nanocomposites have increased one order and decreased two orders of magnitude, respectively. Novel chitosan /oxidized starch /graphene oxide nanocomposites films are prepared in a casting and solvent evaporation method. ${ }^{[53]}$ The tensile strength of these nanocomposites increased as the GO loading increased from 0 to $2.0 \mathrm{wt} \%$. The presence of GO also decreased the MU and increased the degradation temperatures of the nanocomposites. The investigation suggested that the carboxyl groups introduced into the starch and the incorporation of GO can improve the properties of the starch-based composites due to the synergistic interaction and hydrogen bonding between GO, CS, and oxide starch. Starch/lignin biopolymer matrix can also be prepared using graphene oxide as a nanoreinforcing agent. ${ }^{[54]}$ Bionanocomposite films based on starch/lignin blend matrix and GO were prepared by solution-casting technique of the corresponding film-forming solution. The results showed that the content of GO has a significant influence on the mechanical properties of this bionanocomposite and the addition of GO also reduced moisture uptake and water vapor permeability of this bionanocomposite film.

Moreover, starch and modified starch would be used to reduce the graphene oxide. According to Han et al., starch acts as an ideal reductant and stabilizer reduced the graphene oxide with $\mathrm{Ag}\left(\mathrm{NH}_{3}\right)_{2}{ }^{+}$, and $\mathrm{Ag}\left(\mathrm{NH}_{3}\right)_{2}{ }^{+}$can be formed by simultaneous reduction. ${ }^{[55]}$ After reduced by the starch, the $\mathrm{RGO} / \mathrm{Ag}$ NPs showed high stability in aqueous solution. The $\mathrm{RGO} / \mathrm{Ag}$ NPs show higher antimicrobial activity against the gram negative bacterial Pseudomonous aeruginosa than Ag NPs. Because RGO and starch are low-cost and nonpoisonous, the RGO/Ag NPs may have a good prospect as antimicrobial agents for the application in the industry. Potato starch was used as a reductant to reduce graphene oxide. ${ }^{[56]}$ Then the starch reduced graphene oxide was effectively incorporated into PF (phenol formaldehyde) resin by optimizing various processing parameters. The schematic representation of the preparation of $\mathrm{PF} / \mathrm{RGO}$ polymer nanocomposite was shown in Fig. 6. The results confirmed the successful incorporation of starch reduced-GO into the PF matrix, thermal stability of the PF/RGO polymer nanocomposites was increased after the RGO was incorporated into PF resin. The mechanical properties of $\mathrm{PF} / \mathrm{RGO}$ nanocomposites increased with an increase in RGO content up to $0.12 \mathrm{wt} \%$. Moreover, the tensile strength, Young's modulus and impact strength of PF/RGO nanocomposites increase by 56,34 , and $9 \%$ respectively as compared to that of neat PF.

In addition to preparing a film, the compound of starch and graphene can also form hydrogels. Starch-based hydrogels were performed by Diels-Alder cross-linking reactions with improved conducting properties by graphene layers as active nanofillers. ${ }^{[57]}$ After the rGO was added, the nanocomposite hydrogel presented higher mechanical and antimicrobial activity against both gram positive and gram-negative bacteria and the conductivity of the material was improved in a decade. A novel polysaccharide-based nanocomposite hydrogel was obtained from graft copolymerization of acrylamide (AM) monomer onto starch backbones in the presence of graphene oxide (GO) nano sheets and nano-hydroxyapatite to the removal of malachite green (MG) dye from aqueous solution. ${ }^{[58]}$ The process of the nanocomposite adsorbed malachite green was feasible, spontaneous and endothermic. The nanocomposite also has an excellent regeneration capacity after five consecutive cycles of dye adsorptiondesorption.

\section{Summary and Perspective}

The preparation, properties, and applications of starch-based carbonaceous nanofillers are discussed in this review. With the incorporation of these nanofillers, starch-based materials generally show improvement in some of their properties such as mechanical properties, thermal stability and biodegradation rate. The improvement may be due to the homogeneous dispersion of the nanofillers in the matrix and the strong interface adhesion which makes these new materials have a rigid nanofiller network. Moreover, carbonaceous nanofillers have an aspect ratio/surface area, excellent chemical and mechanical properties, which correspondingly influenced the preparation, properties and applications of starch-based carbonaceous nanofillers. However, how the carbonaceous nanofillers affected the crystalline structure and crystallinity of the starch matrix has not been unambiguously elucidated. For better performance and new functionalities to be competitive in the materials world, it is still very important to test new methods and carbonaceous nanofillers to be incorporated with starch for developing promising nanobiocomposites. In addition, the heterogeneous dispersion of the carbonaceous nanofillers in starch issue existed in the past studies should be addressed in the future. Research is also needed regarding the problems with the application and mass production of the starch-based carbonaceous nanofillers. As the nanomaterial and nanotechnology play more and more important role, ${ }^{[59,60]}$ expanding the applications of the carbonbased materials in the other areas would be promising, such as energy related topics. ${ }^{[1,62]}$

\section{Acknowledgments}

We greatly acknowledge the funding for this U. S. Department of Education, Office of Postsecondary Education, Institutional Services (Title III, Part B).

\section{Supporting information}

Not applicable. 


\section{Conflict of interest}

There are no conflicts to declare.

\section{References}

[1] S. Pérez, E. Bertoft, Starch-Stärke, 2010, 62, 389-420, doi 10.1002/star.201000013.

[2] K. Mahmood, H. Kamilah, P.L. Shang, S. Sulaiman, F. Ariffin, A.K. Alias, Food Biosci., 2017, 19, 110-120, doi; 10.1016/j.fbio.2017.05.006.

[3] F. Xie, E. Pollet, P.J. Halley, L. Averous, Prog. Polym. Sci., 2013, 38, 1590-1628, doi: 10.1016/j.progpolymsci.2013.05.002. [4] H. Liu, F. Xie, L. Yu, L. Chen, L. Li, Prog. Polym. Sci., 2009, 34, 1348-1368, doi: 10.1016/j.progpolymsci.2009.07.001.

[5] L. Averous, J. Macromol. Sci. Polym. Rev. Part C: Polym. Rev., 2004, 44, 231-274, doi: 10.1081/MC-200029326.

[6] X.-L. Wang, K.-K. Yang, Y.-Z. Wang, J. Macromol. Sci. Polym. Rev. Part C: Polym. Rev., 2003, 43, 385-409, doi: 10.1081/MC-120023911.

[7] L. Avérous, P. J. Halley, Biofuel Bioprod. Bior., 2009, 3, 329 343, doi: 10.1002/bbb.135.

[8] V. N. Popov, Mater. Sci. Engineer: R: Reports, 2004, 43, 61102, doi: 10.1016/j.mser.2003.10.001.

[9] S. Berber, Y.-K. Kwon, D. Tománek, Phys. Rev. Lett., 2000, 84, 4613, doi: 10.1103/PhysRevLett.84.4613.

[10] A. K. Geim, K. S. Novoselov, The rise of graphene, Nanoscience and technology: a collection of reviews from nature journals, World Scientific, 2010, 11-19.

[11] T. Seyller, A. Bostwick, K. Emtsev, K. Horn, L. Ley, J. McChesney, T. Ohta, J.D. Riley, E. Rotenberg, F. Speck, Phys. Status Solidi B, 2008, 245, 1436-1446, doi: 10.1002/pssb.200844143.

[12] C. N. R. Rao, A. K. Sood, K. S. Subrahmanyam, A. Govindaraj, Angew. Chem. Int. Ed., 2009, 48, 7752-7777, doi 10.1002/anie.200901678.

[13] A. K. Geim, Science, 2009, 324, 1530-1534, doi: 10.1126/science. 1158877 .

[14] M. Pumera, Chem. Rec., 2009, 9, 211-223, doi: 10.1002/tcr.200900008.

[15] A. A. Balandin, S. Ghosh, W. Bao, I. Calizo, D. Teweldebrhan, F. Miao, C.N. Lau, Nano Lett., 2008, 8, 902-907, doi: $10.1021 / \mathrm{nl} 0731872$.

[16] R. F. Service, Science, 2009, 324, 875, doi: 10.1126/science. 324875 .

[17] X. Huang, X. Qi, F. Boey, H. Zhang, Chem. Soc. Rev., 2012, 41, 666-686, doi: 10.1039/C1CS15078B.

[18] N. Soykeabkaew, C. Thanomsilp, O. Suwantong, Compos. Part A Appl. Sci. Manuf., 2015, 78, 246-263, doi: 10.1016/j.compositesa.2015.08.014.

[19] R. Ilyas, S. Sapuan, M. Sanyang, M. Ishak, Nanocrystalline cellulose reinforced starch-based nanocomposite: A review, Conference $5^{\text {th }}$ Postgraduate Seminar on Natural Fiber Composites, 2016, 82-87.

[20] S. Pérez, P.M. Baldwin, D.J. Gallant, Structural features of starch granules I, Starch, Elsevier, 2009, 149-192, doi: 10.1016/B978-0-12-746275-2.00005-7.
[21] J. 1. Jane, Structural features of starch granules II, Starch, Elsevier, 2009, 193-236, doi: 10.1016/B978-0-12-7462752.00006-9.

[22] A. Buléon, P. Colonna, V. Planchot, S. Ball, Int. J. Biol. Macromol. 1998, 23, 85-112, doi: 10.1016/S01418130(98)00040-3.

[23] R. L. Whistler, J. N. BeMiller, E. F. Paschall, Starch: chemistry and technology, Academic Press, 2012.

[24] J. Robin, Cereal Chem., 1974, 51, 389-406.

[25] S. Naguleswaran, T. Vasanthan, R. Hoover, L. Chen, D. Bressler, Food Chem., 2014, 152, 297-299, doi: 10.1016/j.foodchem.2013.11.108.

[26] S. Wang, L. Copeland, Food Funct., 2013, 4, 1564-1580, doi: 10.1039/C3FO60258C.

[27] K. Luengwilai, D.M. Beckles, J. Agric. Food Chem., 2009, 57, 282-291, doi: 10.1021/jf802064w.

[28] S. Wang, J. Yu, J. Yu, Carbohydr. Polym., 2008, 74, 731-739, doi: 10.1016/j.carbpol.2008.03.001.

[29] W. Shujun, Y. Jinglin, L. Hongyan, C. Weiping, Food Chem., 2008, 108, 176-181, doi: 10.1016/j.foodchem.2007.10.059.

[30] V. Varatharajan, R. Hoover, J. Li, T. Vasanthan, K. Nantanga, K. Seetharaman, Q. Liu, E. Donner, S. Jaiswal, R. Chibbar, Food Res. Int., 2011, 44, 2594-2606, doi 10.1016/j.foodres.2011.04.050.

[31] Z. Syahariza, S. Sar, J. Hasjim, M.J. Tizzotti, R.G. Gilbert, Food Chem., 2013, 136, 742-749, doi: 10.1016/j.foodchem.2012.08.053.

[32] J.-1. Jane, J. Appl. Glycosci., 2006, 53, 205-213, doi: 10.5458/jag.53.205.

[33] S. Wang, J. Blazek, E. Gilbert, L. Copeland, Carbohydr. Polym., 2012, 87, 1941-1949, doi: 10.1016/j.carbpol.2011.09.093.

[34] Z. Spitalsky, D. Tasis, K. Papagelis, C. Galiotis, Prog. Polym. Sci., $\quad 2010, \quad 35, \quad 357-401, \quad$ doi: 10.1016/j.progpolymsci.2009.09.003.

[35] J. Cheng, P. Zheng, F. Zhao, X. Ma, Int. J. Biol. Macromol., 2013, 59, 13-19, doi: 10.1016/j.ijbiomac.2013.04.010.

[36] S. K. Swain, A. K. Pradhan, H. S. Sahu, Carbohydr. Polym., 2013, 94, 663-668, doi:10.1016/j.carbpol.2013.01.056.

[37] X. Cao, Y. Chen, P. R. Chang, M. A. Huneault, J. Appl. Polym. Sci., 2007, 106, 1431-1437, doi: 10.1002/app.26799.

[38] L. M. Famá, V. Pettarin, S. N. Goyanes, C. R. Bernal, Carbohydr. Polym., 2011, 83, 1226-1231, doi: 10.1016/j.carbpol.2010.09.027.

[39] L. Yan, P. R. Chang, P. Zheng, Carbohydr. Polym, 2011, 84, 1378-1383, doi: 10.1016/j.carbpol.2011.01.042.

[40] V. Gautam, K.P. Singh, V.L. Yadav, Int. J. Biol. Macromol. 2018, 111, 1124-1132, doi: 10.1016/j.ijbiomac.2018.01.094.

[41] B. Massoumi, P. Jafarpour, M. Jaymand, A.A. Entezami, Polym. Int., 2015, 64, 689-695, doi: 10.1002/pi.4867.

[42] J. Jose, S. De, M. A. A. AlMa'adeed, J. B. Dakua, P. Sreekumar, R. Sougrat, M. A. Al-Harthi, Starch-Stärke, 2015, 67, 147-153, doi: 10.1002/star.201400074.

[43] P. R. Chang, P. Zheng, B. Liu, D. P. Anderson, J. Yu, X. Ma, J. Hazard. Mater, 2011, 186, 2144-2150, doi: 10.1016/j.jhazmat.2010.12.119. 
[44] K. S. Novoselov, A. K. Geim, S.V. Morozov, D. Jiang, Y. Larzek, M. Zahouily, J. Agric. Food Chem., 2017, 65, 10571Zhang, S.V. Dubonos, I.V. Grigorieva, A.A. Firsov, Science, 2004, 10581, doi: 10.1021/acs.jafc.7b04155.

306, 666-669, doi: 10.1126/science.1102896.

[55] Y. Han, Z. Luo, L. Yuwen, J. Tian, X. Zhu, L. Wang, Appl.

[45] M. D. Stoller, S. Park, Y. Zhu, J. An, R. S. Ruoff, Nano Lett., Surf. Sci., 2013, 266, 188-193, doi: 10.1016/j.apsusc.2012.11.132. 2008, 8, 3498-3502, doi:10.1021/n1802558y.

[56] P. Sandhya, M. Sreekala, M. Padmanabhan, K. Jesitha, S.

[46] R. R. Nair, P. Blake, A. N. Grigorenko, K. S. Novoselov, T. Thomas, Compos. $\quad$ B. $\quad$ Eng 2019, 167, 83-92, doi: J. Booth, T. Stauber, N. M. Peres, A. K. Geim, Science, 2008, 320, 10.1016/j.compositesb.2018.12.009.

1308-1308, doi: 10.1126/science.1156965.

[57] K. González, C. García-Astrain, A. Santamaria-Echart, L. [47] T. H. Han, W. J. Lee, D. H. Lee, J. E. Kim, E. Y. Choi, S. O. Ugarte, L. Avérous, A. Eceiza, N. Gabilondo, Carbohydr. Polym., Kim, Adv. Mater, 2010, 22, 2060-2064, doi: 2018, 202, 372-381, doi: 10.1016/j.carbpol.2018.09.007. 10.1002/adma.200903221.

[48] H. Yang, Q. Zhang, C. Shan, F. Li, D. Han, L. Niu, Langmuir, 2010, 26, 6708-6712, doi: 10.1021/la100365z.

[58] H. Hosseinzadeh, S. Ramin, Int. J. Biol. Macromol., 2018, 106, 101-115, doi: 10.1016/j.ijbiomac.2017.07.182.

[59] F. Liu, G. Li, S. Luo, W. Li, Z. Huang, W. Li, F. Su, C. Li, [49] Y. Feng, N. Feng, G. Du, RSC Adv, 2013, 3, 21466-21474, Z. Ding, Q. Jiang, ACS Appl. Mater. Interfaces, 2019, 11, 1691doi: 10.1039/C3RA43025A.

[50] T. Ma, P. R. Chang, P. Zheng, X. Ma, Carbohydr. Polym., 2013, 94, 63-70, doi: 10.1016/j.carbpol.2013.01.007. 1698.

[60] C. Liu, Q. Jiang, Z. Guo, N. L. Lu, M. Mortazavi, NanoScience: Nanomaterial, Nanotechnology and Applications, [51] C. A. Ávila-Orta, F. Soriano Corral, H. A. Fonseca-Florido, F. I. Estrada Aguilar, S. G. Solís Rosales, J. M. Mata Padilla, P. Gonzalez Morones, S. Fernandez Tavizon, E. HernándezHernández, J. Appl. Polym. Sci., 2018, 135, 46037, doi: 10.1002/app.46037.

[52] P. P. Peregrino, M. J. Sales, M. F. da Silva, M. A. Soler, L. F. da Silva, S. G. Moreira, L. G. Paterno, Carbohydr. Polym., 2014, 106, 305-311, doi: 10.1016/j.carbpol.2014.02.008.

[53] J. Ma, C. Liu, R. Li, J. Wang, J. Appl. Polym. Sci., 2012, 123, 2933-2944, doi: 10.1002/app.34901.

[54] M. Aqlil, A. Moussemba Nzenguet, Y. Essamlali, A. Snik, M. $1^{\text {st }}$ edition, 2019, Knoxville, USA, ISBN 978-1-970059-00-72019. [61] Y. Liu, J. Jiang, Y. Yuan, Q. Jiang, C. Yan, Nanomater., 2019, 9, 1336, https://doi.org/10.3390/nano9091336.

[62] Z. Peng, Y. Hu, Ji. Wang, S. Liu, C. Li, Q. Jiang, J. Lu, X. Zeng, P. Peng, F. Li, Adv. Energy Mater., 2019, 1802928, doi: 10.1002/aenm.201802928.

Publisher's Note: Engineered Science Publisher remains neutral with regard to jurisdictional claims in published maps and institutional affiliations. 